\title{
Finite-size corrections for the attractive mean-field monomer-dimer model
}

\author{
Diego Alberici ${ }^{1} \quad$ Pierluigi Contucci $^{1} \quad$ Rachele Luzi $^{1}$ \\ Cecilia Vernia ${ }^{2}$ \\ ${ }^{1}$ Dipartimento di Matematica, Università di Bologna, Italy \\ ${ }^{2}$ Dipartimento di Scienze Fisiche Informatiche e Matematiche, Università di Modena \\ e Reggio Emilia, Italy
}

August 9, 2021

\begin{abstract}
The finite volume correction for a mean-field monomer-dimer system with an attractive interaction are computed for the pressure density, the monomer density and the susceptibility. The results are obtained by introducing a two-dimensional integral representation for the partition function decoupling both the hard-core interaction and the attractive one. The next-to-leading terms for each of the mentioned quantities is explicitly derived as well as the value of their sign that is related to their monotonic convergence in the thermodynamic limit.
\end{abstract}

\section{Introduction}

Monomer-dimer systems are known to have no phase transitions when the only interaction is the hard-core one. This fact has been rigorously proved by Heilmann and Lieb 22 23]. If instead an attractive interaction is present, favouring configurations where similar particles sit in neighbouring sites, a phase transition may be expected and has been studied for some finite-dimensional cases [1,24,25] and in the mean-field setting [3,4, later developed and applied in 6, 7, 13, 16, 27. Monomer-dimer models are also related to the matching problem in computer science, where the statistical mechanics approach has conveyed important results [2, 11,29.

In the present paper we continue the investigation of the mean-field case by controlling the finite-size corrections of the main thermodynamic quantities describing the model, namely the pressure density, the monomer density and the susceptibility. This is relevant in Statistical Mechanics and its applications $8-10$ because the size and the sign of the corrections carry some important information on the phase transition within the phase space.

More precisely, for instance for the pressure, we are interested in proving the existence of the limit and the relative properties for the next-to- 
leading term:

$$
\Lambda_{N}=\log Z_{N}-N p_{*},
$$

where $p_{*}$ is the pressure density in the thermodynamic limit. Among the informations that $\Lambda_{N}$ carries, its sign is related to the type of monotonic behaviour for large $N$, namely whether the pressure reaches its limit from above or below, and is therefore important to understand whether the finite volume approximation is by excess or defect (see [16 for its relevance in the inverse problem). We notice that while for the systems in finite-dimensional lattices the next to leading terms identify surface contributions and further sub-leading powers of the linear size (see for instance for the ferromagnetic Ising model [19] and spin-glass Edward-Anderson model [17), in the mean-field case the first correction is of order one. In finite-dimensional lattices moreover the sign of the next to leading terms are related to local correlation inequalities (see [20, 21, 26] for the ferromagnets and 18] for the spin-glass Edwards-Anderson), while sometimes in the mean field case there are global positivity properties that leads to positivity [14]. In our case the presence of two interactions of different nature, the repulsive hard-core that forbids the overlap of two particles in the same site and the attraction that favours the closeness of similar articles, makes the identification of the next to leading term particularly challenging. To this purpose we introduce a new technical tool, a twodimensional transform able to decouple, separately, the two interactions. Like in the case of the Laplace transform in standard ferromagnets this enable us to obtain explicit expression in terms of the solution of the model and evaluate the sign of each correction.

\section{Definitions and results}

Consider the complete graph of size $N$. A monomer-dimer configuration $D$ on the set of vertices $V_{N}=\{1, \ldots, N\}$ is a partition into pairs of a subset $A \subset V_{N}$ : the pairs $\{i, j\} \in D$ are called dimers, while the vertices $i \in V_{N} \backslash A$ are called monomers. We denote the monomer density by

$$
m_{N}(D)=\frac{1}{N}\left|V_{N} \backslash A\right|=\frac{N-2|D|}{N} .
$$

Beyond the hard-core interaction (two dimers cannot overlap), we consider also a mean-field attractive interaction and we define the following Hamiltonian:

$$
H_{N}(D)=-N\left(\frac{a}{2} m_{N}(D)^{2}+b m_{N}(D)\right)
$$

with parameters $a>0$ and $b \in \mathbb{R}$. Denoting by $\mathscr{D}_{N}$ the configuration space, the partition function of the system is

$$
Z_{N}=\sum_{D \in \mathscr{D}_{N}} N^{-|D|} e^{-H_{N}(D)} .
$$

We denote by $\langle\cdot\rangle_{N}$ the expected value with respect to the associated Gibbs measure, namely for any observable $f: \mathscr{D}_{N} \rightarrow \mathbb{R}$,

$$
\langle f\rangle_{N}=\frac{1}{Z_{N}} \sum_{D \in \mathscr{D}_{N}} f(D) N^{-|D|} e^{-H_{N}(D)}
$$


It is worth remarking that this model coincides with that studied in [3], by the change of parameters

$$
a=2 J, \quad b=h-J .
$$

Theorem 1 (Finite-size corrections). Let $a>0, b \in \mathbb{R}$ such that the system has a unique phase (see [3] for the coexistence line). The pressure density of a system of size $N$ is

$$
p_{N}:=\frac{1}{N} \log Z_{N}=p_{*}+\frac{\Lambda}{N}+O\left(\frac{1}{N^{2}}\right),
$$

the average monomer density is

$$
\mu_{N}:=\left\langle m_{N}\right\rangle_{N}=m_{*}+\frac{\Lambda^{\prime}}{N}+O\left(\frac{1}{N^{2}}\right),
$$

and the susceptibility is

$$
\chi_{N}:=N\left(\left\langle m_{N}^{2}\right\rangle_{N}-\left\langle m_{N}\right\rangle_{N}^{2}\right)=\chi_{*}+\frac{\Lambda^{\prime \prime}}{N}+O\left(\frac{1}{N^{2}}\right) .
$$

$p_{*}, m_{*}, \chi_{*}, \Lambda, \Lambda^{\prime}, \Lambda^{\prime \prime}$ depend on the parameters $a, b$, but not on the size $N$. Their expressions rely on the implicit expression for the limiting monomer density $m_{*}=y_{*}$ (see the self-consistent equation (27) or, equivalently refer to [3]). In terms of $F(x, y), D, \mathcal{L}, \mathcal{K}_{G}, \mathcal{M}_{G}$ that will be defined precisely in Section 4 (equations (25), (34), (39), (41), (43) respectively), we have:

$$
\begin{aligned}
& p_{*}=F\left(\sqrt{1-m_{*}}, m_{*}\right) \\
& \Lambda=-\log \sqrt{\frac{D}{a}} \\
& \Lambda^{\prime}=\mathcal{K}_{g} \\
& \chi_{*}=m_{*}\left(1-m_{*}\right)+\mathcal{K}_{\left(g-m_{*}\right)^{2}}^{(1)} \\
& \Lambda^{\prime \prime}=\mathcal{K}_{g(1-g)}-\mathcal{K}_{\left(g-m_{*}\right)^{2}}^{(1)} \mathcal{L}+\mathcal{M}_{\left(g-m_{*}\right)^{2}}-\left(\mathcal{K}_{g}\right)^{2}
\end{aligned}
$$

The computation of the finite size corrections relies on the following integral representation, which decouples both the attractive interaction and the hard-core interaction.

Proposition 1 (Integral representation). For any $a>0, b \in \mathbb{R}$, the partition function admits the following integral representation:

$$
Z_{N}=\frac{N \sqrt{a}}{2 \pi} \iint_{\mathbb{R}^{2}} \Phi(x, y)^{N} \mathrm{~d} x \mathrm{~d} y
$$

where

$$
\Phi(x, y)=\left(x+e^{a y+b}\right) \exp \left(-\frac{x^{2}}{2}-a \frac{y^{2}}{2}\right) .
$$

The previous integral representation is based on two properties of Gaussian measures, that we recall in the next lemmas. 
Lemma 1 (Hubbart-Stratonovich transform, or the Gaussian moments generating function). For any $\sigma>0$ and $t \in \mathbb{R}$,

$$
\exp \left(\frac{t^{2} \sigma^{2}}{2}\right)=\frac{1}{\sqrt{2 \pi \sigma^{2}}} \int_{\mathbb{R}} \exp \left(t y-\frac{y^{2}}{2 \sigma^{2}}\right) \mathrm{d} y
$$

Lemma 2 (Wick-Isserlis rule for the Gaussian moments). For any $\sigma>0$ and any finite set $A$,

$$
\sum_{D \text { partition of } A \text { into pairs }} \sigma^{2|D|}=\frac{1}{\sqrt{2 \pi \sigma^{2}}} \int_{\mathbb{R}} x^{|A|} \exp \left(-\frac{x^{2}}{2 \sigma^{2}}\right) \mathrm{d} x
$$

Proof of Proposition 1 First we use the Hubbart-Stratonovich transform to decouple the attractive interaction. Choosing $t=N a m_{N}(D)$ and $\sigma^{2}=\frac{1}{N a}$ in (17), the partition function (4) rewrites as

$$
\begin{aligned}
Z_{N}(a, b) & =\sum_{D \in \mathscr{D}_{N}} N^{-|D|} \exp N\left(\frac{a}{2} m_{N}(D)^{2}+b m_{N}(D)\right)= \\
& =\sum_{D \in \mathscr{D}_{N}} N^{-|D|} \sqrt{\frac{N a}{2 \pi}} \int_{\mathbb{R}} \exp N\left((a y+b) m_{N}(D)-\frac{a y^{2}}{2}\right) \mathrm{d} y= \\
& =\sqrt{\frac{N a}{2 \pi}} \int_{\mathbb{R}} Z_{N}^{(0)}(a y+b) \exp \left(-N \frac{a y^{2}}{2}\right) \mathrm{d} y
\end{aligned}
$$

where $Z_{N}^{(0)}\left(b^{\prime}\right)$ denotes the partition function $Z_{N}\left(a=0, b=b^{\prime}\right)$ of the model without attractive interaction. Now we use the Wick-Isserlis rule to decouple the hard-core interaction, as was shown in [5, 28. Choosing $\sigma^{2}=N^{-1}$ and $A \subseteq V_{N}$ in (18), the non-attractive partition function rewrites as

$$
\begin{aligned}
Z_{N}^{(0)}\left(b^{\prime}\right) & =\sum_{D \in \mathscr{D}_{N}} N^{-|D|} \exp \left(b^{\prime} N m_{N}(D)\right)= \\
& =\sum_{A \subseteq V_{N}} e^{b^{\prime}(N-|A|)} \sqrt{\frac{N}{2 \pi}} \int_{\mathbb{R}} x^{|A|} \exp \left(-N \frac{x^{2}}{2}\right) \mathrm{d} x= \\
& =\sqrt{\frac{N}{2 \pi}} \int_{\mathbb{R}}\left(x+e^{b^{\prime}}\right)^{N} \exp \left(-N \frac{x^{2}}{2}\right) \mathrm{d} x .
\end{aligned}
$$

Substituting (20) with $b^{\prime}=a y+b$ into (19), we finally obtain (15).

\section{Monotonicity regions}

The finite-size corrections for the pressure density $p_{N}$, the average monomer density $\mu_{N}$ and the susceptibility $\chi_{N}$ can be used to determine the monotonicity of the three sequences with respect to the size of the system $N$. To be precise, the signs of the corrections $\Lambda, \Lambda^{\prime}, \Lambda^{\prime \prime}$ in Theorem 1 determine whether $p_{N}, \mu_{N}, \chi_{N}$ reach their respective limits $p_{*}, m_{*}, \chi_{*}$ from above or from below. Figure 1 shows the phase space regions where $\Lambda$ (green curve), $\Lambda^{\prime}$ (red curve) and $\Lambda^{\prime \prime}$ (blue curve) change sign. 


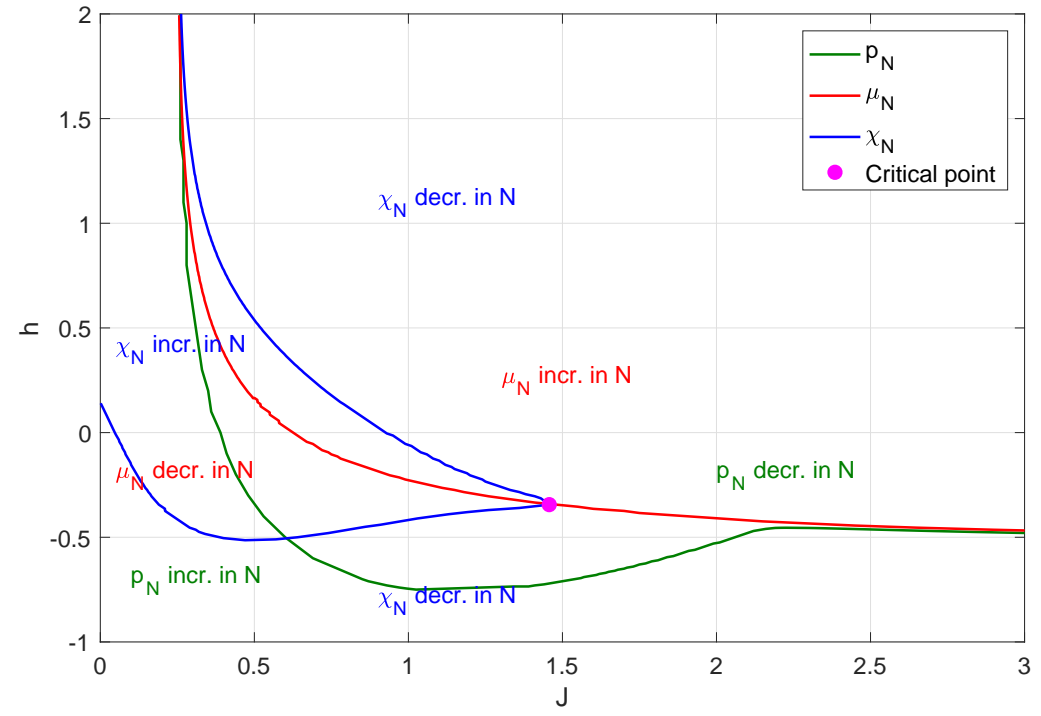

Figure 1: Phase space $(J, h)$. The green curve represents the values $(J, h)$ for which the pressure density $p_{N}$ changes monotonicity with respect to $N$ (i.e. $\Lambda$ changes sign). The red curve represents the values $(J, h)$ for which the average monomer density $\mu_{N}$ changes monotonicity with respect to $N$ (i.e. $\Lambda^{\prime}$ changes sign). The blue curve represents the values $(J, h)$ for which the susceptibility $\chi_{N}$ changes monotonicity with respect to $N$ (i.e. $\Lambda^{\prime \prime}$ changes sign). The purple dot is the critical point of the system. 

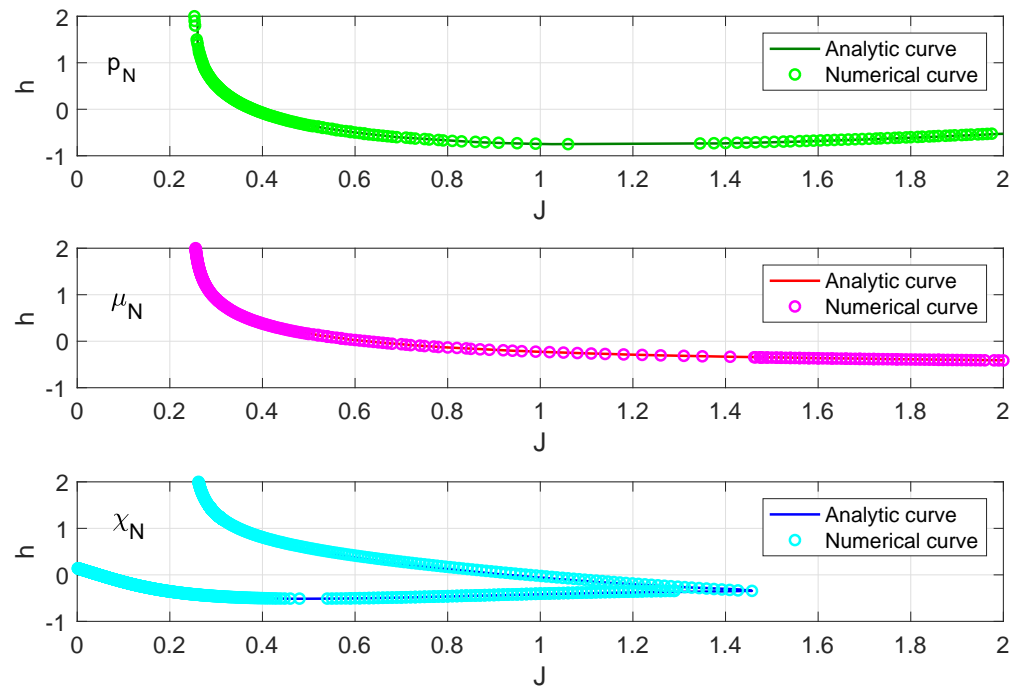

Figure 2: Phase space $(J, h)$ : comparison between analytical and numerical monotonicity (with respect to $N$ ) curves for the pressure density $p_{N}$ (upper panel), for the average monomer density $\mu_{N}$ (middle panel) and for the susceptibility $\chi_{N}$ (lower panel). The analytical curves (continuous lines) are the same as in Fig. 1]

Due to the mean field nature of the model, the Gibbs measure and the expected value with respect to such measure at finite volume size $N$ can be computed by evaluating the combinatorial weights of the possible dimer density values, that is the number of the possible configurations that share the same value $|D|$ of dimers on the complete graph with $N$ vertices [16. This enable us to compute numerically the phase space curves in which $p_{N}, \mu_{N}$ and $\chi_{N}$ invert the monotonicity with respect to $N$. The comparison of these numerical curves with the ones obtained analitically from $\Lambda, \Lambda^{\prime}$ and $\Lambda^{\prime \prime}$ (Fig. 1) is shown in Fig. 2. The perfect overlap between the curves is evident.

Remark 1. It is possible to modify the Hamiltonian of the system in such a way that the Gibbs measure does not change, but the new pressure density $\tilde{p}_{N}$ reaches its limit $p_{*}$ from above as $N \rightarrow \infty$ in the whole phase space $(a, b)$. Namely

$$
\lim _{N \rightarrow \infty} \tilde{p}_{N}=\inf _{N} \tilde{p}_{N} \quad \forall a \geq 0, b \in \mathbb{R} .
$$

The finite size correction $\Lambda=-\log \sqrt{D / a}$ has range $(-\log \sqrt{2},+\infty)$, indeed it is easy to compute explicitly the determinant

$$
\frac{D}{a}=2-y_{*}-2 a y_{*}\left(1-y_{*}\right)
$$


which is non-negative by definition, takes value 0 at the critical point of the system and goes to 2 as $b \rightarrow-\infty$ and $a$ is fixed. Therefore it suffices to set $\tilde{H}_{N}(D)=H_{N}(D)-\log \sqrt{2}$ for all configurations $D \in \mathscr{D}_{N}$ in order to obtain

$$
\tilde{p}_{N}=p_{N}+\frac{\log \sqrt{2}}{N}=p_{*}+\frac{\tilde{\Lambda}}{N}+O\left(\frac{1}{N^{2}}\right)
$$

where $\tilde{\Lambda}=\Lambda+\log \sqrt{2}>0$ for all $a \geq 0, b \in \mathbb{R}$.

On the contrary it is not possible to obtain a modified pressure density reaching $p_{*}$ from below in the whole phase space, since the upper bound of the finite size correction $\Lambda$ is $+\infty$.

\section{Computation of the finite-size correc- tions}

The integral representation (15) allows to compute the finite-size corrections by estimations of suitable Laplace two-dimensional integrals [12]. Laplace estimates are needed up to order $N^{-1}$ in the case of the magnetisation and up to order $N^{-2}$ in the case of the susceptibility.

We denote by $\left(x_{*}, y_{*}\right)$ the global maximum of $\Phi$ on $\mathbb{R}^{2}$. First of all, we observe that $x_{*}, y_{*}>0$ since

$$
\Phi\left(s_{1} x, s_{2} y\right) \leq \Phi(x, y) \quad \forall x, y>0 \quad \forall s_{1}, s_{2}= \pm 1
$$

and the inequality is strict if $s_{1}, s_{2}$ are not both 1 . It is convenient to set for $x, y>0$

$$
F(x, y):=\log \Phi(x, y)=-\frac{x^{2}}{2}-a \frac{y^{2}}{2}+\log \left(x+e^{a y+b}\right) .
$$

The condition $\nabla F\left(x_{*}, y_{*}\right)=0$ says that $\left(x_{*}, y_{*}\right)$ is a solution of the following fixed point system:

$$
\left\{\begin{array}{l}
x=\frac{1}{x+e^{a y+b}} \\
y=\frac{e^{a y+b}}{x+e^{a y+b}}
\end{array}\right.
$$

which rewrites as:

$$
\left\{\begin{array}{l}
x=\sqrt{1-y} \\
e^{a y+b}=\frac{y}{\sqrt{1-y}}
\end{array} .\right.
$$

The second equation in (27) is self-consistent and an elementary analysis shows that it has a unique solution $y_{*}$ for $a \leq a_{c}:=(3+2 \sqrt{2}) / 2$. For $a>a_{c}$ there are at most 3 solutions, one in each of the following intervals $\left(0, y_{-}\right),\left(y_{-}, y_{+}\right),\left(y_{+}, 1\right)$, where $y_{ \pm}:=\left(2 a+1 \pm \sqrt{4 a^{2}-12 a+1}\right) /(4 a)$; $y_{*}$ is the solution maximizing $F\left(x_{*}, y_{*}\right)$ with $x_{*}=\sqrt{1-y_{*}} . y_{*}$ will be two-values only for parameters $a, b$ on the coexistence line [3], a case that we exclude from the present paper for the sake of simplicity. 
Now set also

$$
g(x, y):=\frac{\partial F}{\partial b}(x, y)=\frac{e^{a y+b}}{x+e^{a y+b}} .
$$

The average monomer density, using the integral representation (15) for the partition function, rewrites as

$$
\mu_{N}=\frac{1}{N} \frac{\partial}{\partial b} \log Z_{N}=g\left(x_{*}, y_{*}\right)+\frac{\iint \Phi(x, y)^{N}\left(g(x, y)-g\left(x_{*}, y_{*}\right)\right) \mathrm{d} x \mathrm{~d} y}{\iint \Phi(x, y)^{N} \mathrm{~d} x \mathrm{~d} y}
$$

The susceptibility rewrites as

$$
\begin{aligned}
\chi_{N}= & \frac{1}{N} \frac{\partial^{2}}{\partial b^{2}} \log Z_{N}= \\
= & N \frac{\iint \Phi(x, y)^{N}\left(g(x, y)-g\left(x_{*}, y_{*}\right)\right)^{2} \mathrm{~d} x \mathrm{~d} y}{\iint \Phi(x, y)^{N} \mathrm{~d} x \mathrm{~d} y}+ \\
& -N\left(\frac{\iint \Phi(x, y)^{N}\left(g(x, y)-g\left(x_{*}, y_{*}\right)\right) \mathrm{d} x \mathrm{~d} y}{\iint \Phi(x, y)^{N} \mathrm{~d} x \mathrm{~d} y}\right)^{2}+ \\
& +\frac{\iint \Phi(x, y)^{N} g(x, y)(1-g(x, y)) \mathrm{d} x \mathrm{~d} y}{\iint \Phi(x, y)^{N} \mathrm{~d} x \mathrm{~d} y}
\end{aligned}
$$

Both in (29) and (30), the term $g\left(x_{*}, y_{*}\right)$ has been artificially introduced in order to simplify the following computations. By the way, observe that according to (26), $g\left(x_{*}, y_{*}\right)=y_{*}$.

Expressions (29) and (30) can be typically approximated by the Laplace method. These estimates involve Gaussian moments and higher order derivatives of $F$ and $g$ at the maximum point $\left(x_{*}, y_{*}\right)$ of $F$. Therefore it will be convenient to introduce the following notations:

$$
F_{i, j}:=\frac{\partial^{i+j} F}{\partial x^{i} \partial y^{j}}\left(x_{*}, y_{*}\right)
$$

while

$$
\phi_{i, j}:=\frac{1}{2 \pi} \iint_{\mathbb{R}^{2}} x^{i} y^{j} \exp \left(-\frac{1}{2}(x, y) C(x, y)^{T}\right) \mathrm{d} x \mathrm{~d} y
$$

where

$$
\begin{gathered}
C:=\left(-\operatorname{Hess} F\left(x_{*}, y_{*}\right)\right)^{-1}=\frac{1}{D}\left(\begin{array}{cc}
-F_{0,2} & F_{1,1} \\
F_{1,1} & -F_{2,0}
\end{array}\right), \\
D:=\operatorname{det}\left(-\operatorname{Hess} F\left(x_{*}, y_{*}\right)\right)=F_{0,2} F_{2,0}-F_{1,1}^{2} .
\end{gathered}
$$

Proposition 2 (Laplace estimates). Consider the integral

$$
I_{N}(G):=\iint_{\mathbb{R}^{2}} \Phi(x, y)^{N} G(x, y) \mathrm{d} x \mathrm{~d} y
$$


where $G$ is any real function, analytic in a neighbourhood of $\left(x_{*}, y_{*}\right)$. Set

$$
I_{N}^{\prime}(G):=I_{N}(G) /\left(\frac{2 \pi}{N \sqrt{D}} e^{N F\left(x_{*}, y_{*}\right)}\right) .
$$

Provided that the global maximum point $\left(x_{*}, y_{*}\right)$ is unique and the Hessian matrix $-C^{-1}$ is negative definite, the following estimates hold true:

a)

$$
I_{N}^{\prime}(G)=G\left(x_{*}, y_{*}\right)+O\left(N^{-1}\right)
$$

b) If $G(x, y) \equiv 1$ for all $(x, y) \in \mathbb{R}^{2}$,

$$
I_{N}^{\prime}(G)=1+\mathcal{L} N^{-1}+O\left(N^{-2}\right)
$$

where

$$
\begin{aligned}
& \mathcal{L}=\mathcal{L}^{(1)}+\mathcal{L}^{(2)}, \\
& \mathcal{L}^{(1)}=\sum_{i+j=4} \frac{F_{i, j}}{i ! j !} \frac{\phi_{i, j}}{D} \\
& \mathcal{L}^{(2)}=\sum_{\substack{i_{1}+j_{1}=3, i_{2}+j_{2}=3}} \frac{F_{i_{1}, j_{1}}}{i_{1} ! j_{1} !} \frac{F_{i_{2}, j_{2}}}{i_{2} ! j_{2} !} \frac{\phi_{i_{1}+i_{2}, j_{1}+j_{2}}}{D^{2}}
\end{aligned}
$$

c) If $G\left(x_{*}, y_{*}\right)=0$,

$$
I_{N}^{\prime}(G)=\mathcal{K}_{G} N^{-1}+O\left(N^{-2}\right),
$$

where

$$
\begin{aligned}
\mathcal{K}_{G} & =\mathcal{K}_{G}^{(1)}+\mathcal{K}_{G}^{(2)} \\
\mathcal{K}_{G}^{(1)} & =\sum_{i+j=2} \frac{G_{i, j}}{i ! j !} \frac{\phi_{i, j}}{D} \\
\mathcal{K}_{G}^{(2)} & =\sum_{\substack{i_{1}+j_{1}=3 \\
i_{2}+j_{2}=1}} \frac{F_{i_{1}, j_{1}}}{i_{1} ! j_{1} !} \frac{G_{i_{2}, j_{2}}}{i_{2} ! j_{2} !} \frac{\phi_{i_{1}+i_{2}, j_{1}+j_{2}}}{D^{2}}
\end{aligned}
$$

d) If $G\left(x_{*}, y_{*}\right)=0$ and $\nabla G\left(x_{*}, y_{*}\right)=0$,

$$
I_{N}^{\prime}(G)=\mathcal{K}_{G}^{(1)} N^{-1}+\mathcal{M}_{G} N^{-2}+O\left(N^{-3}\right),
$$

where

$$
\begin{aligned}
& \mathcal{M}_{G}= \mathcal{M}_{G}^{(1)}+\mathcal{M}_{G}^{(2)}+\mathcal{M}_{G}^{(3)}+\mathcal{M}_{G}^{(4)} \\
& \mathcal{M}_{G}^{(1)}=\sum_{i+j=4} \frac{G_{i, j}}{i ! j !} \frac{\phi_{i, j}}{D^{2}} \\
& \mathcal{M}_{G}^{(2)}=\sum_{\substack{i_{1}+j_{1}=3, i_{2}+j_{2}=3}} \frac{F_{i_{1}, j_{1}}}{i_{1} ! j_{1} !} \frac{G_{i_{2}, j_{2}}}{i_{2} ! j_{2} !} \frac{\phi_{i_{1}+i_{2}, j_{1}+j_{2}}}{D^{3}} \\
& \mathcal{M}_{G}^{(3)}=\sum_{\substack{i_{1}+j_{1}=4, i_{2}+j_{2}=2}} \frac{F_{i_{1}, j_{1}}}{i_{1} ! j_{1} !} \frac{G_{i_{2}, j_{2}}}{i_{2} ! j_{2} !} \frac{\phi_{i_{1}+i_{2}, j_{1}+j_{2}}}{D^{3}} \\
& \mathcal{M}_{G}^{(4)}=\frac{1}{2} \sum_{\substack{i_{1}+j_{1}=3, i_{2}+j_{2}=3, i_{3}+j_{3}=2}} \frac{F_{i_{1}, j_{1}}}{i_{1} ! j_{1} !} \frac{F_{i_{2}, j_{2}} !}{i_{2} ! j_{2} !} \frac{G_{i_{3}, j_{3}} !}{i_{3} ! j_{3} !} \frac{\phi_{i_{1}+i_{2}+i_{3}, j_{1}+j_{2}+j_{3}}}{D^{4}} .
\end{aligned}
$$


Sketch of the Proof. Since $\Phi(x, y)$ takes its maximum on $(0,1)^{2}$, any contribution to the integral $I_{N}$ coming from $(x, y) \in \mathbb{R}^{2} \backslash(0,1)^{2}$ is exponentially small compared to the contribution given by $(x, y) \in(0,1)^{2}$. We write $I_{N}^{\prime} \approx J_{N}^{\prime}$ if there exists $\delta>0$ such that $I_{N}^{\prime}=J_{N}^{\prime}+O\left(e^{-\delta N}\right)$ for every $N$. Observe that

$$
I_{N}^{\prime} \approx \frac{N \sqrt{D}}{2 \pi} \int_{0}^{1} \int_{0}^{1} \exp N\left(F(x, y)-F\left(x_{*}, y_{*}\right)\right) G(x, y) \mathrm{d} x \mathrm{~d} y .
$$

Then make the change of variable $(x, y) \mapsto\left(x_{*}, y_{*}\right)+\frac{1}{\sqrt{N}}(x, y)$ and expand $F$ around $\left(x_{*}, y_{*}\right)$. Since $\nabla F\left(x_{*}, y_{*}\right)=0$, one obtains that

$$
I_{N}^{\prime} \approx \frac{\sqrt{D}}{2 \pi} \iint_{A_{N}} \exp \left(-\frac{1}{2}(x, y) C^{-1}(x, y)^{T}\right) e^{f_{N}(x, y)} G_{N}(x, y) \mathrm{d} x \mathrm{~d} y,
$$

where $A_{N}:=\left(-x_{*} \sqrt{N},\left(1-x_{*}\right) \sqrt{N}\right) \times\left(-y_{*} \sqrt{N},\left(1-y_{*}\right) \sqrt{N}\right)$ and

$$
\begin{aligned}
f_{N}(x, y) & :=\sum_{i+j \geq 3} \frac{F_{i, j}}{i ! j !} x^{i} y^{j} N^{-\frac{i+j}{2}+1}, \\
G_{N}(x, y) & :=\sum_{i+j \geq 0} \frac{G_{i, j}}{i ! j !} x^{i} y^{j} N^{-\frac{i+j}{2}} .
\end{aligned}
$$

Let $(X, Y)$ be a centred Gaussian vector with covariance matrix $C$ : 455) rewrites as

$$
I_{N}^{\prime} \approx \mathbb{E}\left[e^{f_{N}(X, Y)} G_{N}(X, Y) \mathbb{1}\left((X, Y) \in A_{N}\right)\right] .
$$

We remark that there exist $K, \delta>0$ such that $\mathbb{P}\left((X, Y) \notin A_{N}\right) \leq K e^{-\delta N}$ for all $N$. Therefore the orders $1, N^{-1}, N^{-2}, \ldots$ of $I_{N}^{\prime}$ are obtained by multiplying the suitable terms in the Taylor expansions of $e^{f_{N}}=1+f_{N}+$ $\frac{1}{2} f_{N}^{2}+\ldots$ and $G_{N}$ and computing the corresponding Gaussian moments of $(X, Y)$. It is worth noticing that the fractional orders $N^{-\frac{1}{2}}, N^{-\frac{3}{2}}, \ldots$. are zero because the odd Gaussian moments are zero.

The integral representations of the pressure density (15), the average monomer density (29) and the susceptibility (30) can be estimated according to Proposition 2 yielding a proof of Theorem 1 In the Appendix we compute explicitly the Gaussian moments and the derivatives that appear in the expressions of the finite-size corrections.

Remark 2. An elementary computation shows that the susceptibility limit $\chi_{*}$ found in the present paper (13) coincides with that obtained in [16] (equation 5) by direct differentiation of the consistency equation, namely

$$
\chi_{*}=\frac{2 m_{*}\left(1-m_{*}\right)}{2-m_{*}-2 a m_{*}\left(1-m_{*}\right)} .
$$




\section{Appendix}

The even moments of $(X, Y)$, centered Gaussian vector of covariance matrix (33), are computed up to order 8 using the Wick's rule:

$$
\begin{aligned}
& \phi_{2,0}=-F_{0,2}, \quad \phi_{1,1}=F_{1,1}, \quad \phi_{0,2}=-F_{2,0} \\
& \phi_{4,0}=3 F_{0,2}^{2}, \quad \phi_{3,1}=-3 F_{0,2} F_{1,1} \\
& \phi_{2,2}=F_{0,2} F_{2,0}+2 F_{1,1}^{2} \\
& \phi_{1,3}=-3 F_{1,1} F_{2,0}, \quad \phi_{0,4}=3 F_{2,0}^{2} \\
& \phi_{6,0}=-15 F_{0,2}^{3}, \quad \phi_{5,1}=15 F_{0,2}^{2} F_{1,1} \\
& \phi_{4,2}=-3 F_{0,2}^{2} F_{2,0}-12 F_{0,2} F_{1,1}^{2} \\
& \phi_{3,3}=9 F_{0,2} F_{1,1} F_{2,0}+6 F_{1,1}^{3} \\
& \phi_{2,4}=-3 F_{0,2} F_{2,0}^{2}-12 F_{1,1}^{2} F_{2,0}, \\
& \phi_{1,5}=15 F_{1,1} F_{2,0}^{2}, \quad \phi_{0,6}=-15 F_{2,0}^{3}, \\
& \phi_{8,0}=105 F_{0,2}^{4}, \quad \phi_{7,1}=-105 F_{0,2}^{3} F_{1,1}, \\
& \phi_{6,2}=90 F_{0,2}^{2} F_{1,1}^{2}+15 F_{0,2}^{3} F_{2,0}, \\
& \phi_{5,3}=-45 F_{0,2}^{2} F_{1,1} F_{2,0}-60 F_{2,0} F_{1,1}^{3}, \\
& \phi_{4,4}=9 F_{0,2}^{2} F_{2,0}^{2}+24 F_{1,1}^{4}+72 F_{0,2} F_{1,1}^{2} F_{2,0}, \\
& \phi_{3,5}=-45 F_{0,2} F_{1,1} F_{2,0}^{2}-60 F_{0,2} F_{1,1}^{3}, \\
& \phi_{2,6}=90 F_{1,1}^{2} F_{2,0}^{2}+15 F_{0,2} F_{2,0}^{3}, \\
& \phi_{1,7}=-105 F_{2,0}^{3} F_{1,1}, \quad \phi_{0,8}=105 F_{2,0}^{4} .
\end{aligned}
$$

The derivatives of $F$ at its maximum point $\left(x_{*}, y_{*}\right)$ up to order 4 , in terms of $y_{*}$ only are:

$$
\begin{aligned}
& F_{2,0}=-2+y_{*}, \quad F_{1,1}=-a y_{*} \sqrt{1-y_{*}}, \\
& F_{0,2}=-a+a^{2} y_{*}\left(1-y_{*}\right), \\
& F_{3,0}=2\left(1-y_{*}\right)^{3 / 2}, \quad F_{2,1}=2 a y_{*}\left(1-y_{*}\right), \\
& F_{1,2}=-a^{2} y_{*} \sqrt{1-y_{*}}\left(1-2 y_{*}\right), \quad F_{0,3}=a^{3} y_{*}\left(1-y_{*}\right)\left(1-2 y_{*}\right), \\
& F_{4,0}=-6\left(1-y_{*}\right)^{2}, \quad F_{3,1}=-6 a y_{*}\left(1-y_{*}\right)^{3 / 2}, \\
& F_{2,2}=2 a^{2} y_{*}\left(1-y_{*}\right)\left(1-3 y_{*}\right), \\
& F_{1,3}=-a^{3} y_{*} \sqrt{1-y_{*}}\left(1-6 y_{*}+6 y_{*}^{2}\right), \\
& F_{0,4}=a^{4} y_{*}\left(1-y_{*}\right)\left(1-6 y_{*}+6 y_{*}^{2}\right) .
\end{aligned}
$$

The derivatives of $g$ at $\left(x_{*}, y_{*}\right)$ up to order 3 :

$$
\begin{aligned}
& g_{1,0}=-y_{*} \sqrt{1-y_{*}}, \quad g_{0,1}=a y_{*}\left(1-y_{*}\right), \quad g_{2,0}=2 y_{*}\left(1-y_{*}\right), \\
& g_{1,1}=-a y_{*} \sqrt{1-y_{*}}\left(1-2 y_{*}\right), \quad g_{0,2}=a^{2} y_{*}\left(1-y_{*}\right)\left(1-2 y_{*}\right), \\
& g_{3,0}=-6 y_{*}\left(1-y_{*}\right)^{3 / 2}, \quad g_{2,1}=2 a y_{*}\left(1-y_{*}\right)\left(1-3 y_{*}\right), \\
& g_{1,2}=-a^{2} y_{*} \sqrt{1-y_{*}}\left(1-6 y_{*}+6 y_{*}^{2}\right) \\
& g_{0,3}=a^{3} y_{*}\left(1-y_{*}\right)\left(1-6 y_{*}+6 y_{*}^{2}\right) .
\end{aligned}
$$


The derivatives of $\tilde{g}:=\left(g-y_{*}\right)^{2}$ at $\left(x_{*}, y_{*}\right)$ up to order 4 :

$$
\begin{aligned}
& \tilde{g}_{2,0}=2 g_{1,0}^{2}, \quad \tilde{g}_{1,1}=2 g_{1,0} g_{0,1}, \quad \tilde{g}_{0,2}=2 g_{0,1}^{2}, \\
& \tilde{g}_{3,0}=6 g_{1,0} g_{2,0}, \quad \tilde{g}_{2,1}=4 g_{1,0} g_{1,1}+2 g_{0,1} g_{2,0}, \\
& \tilde{g}_{1,2}=4 g_{0,1} g_{1,1}+2 g_{1,0} g_{0,2}, \quad \tilde{g}_{0,3}=6 g_{0,1} g_{0,2}, \\
& \tilde{g}_{4,0}=6 g_{2,0}^{2}+8 g_{1,0} g_{3,0}, \\
& \tilde{g}_{3,1}=6 g_{2,0} g_{1,1}+6 g_{1,0} g_{2,1}+2 g_{0,1} g_{3,0}, \\
& \tilde{g}_{2,2}=4 g_{1,1}^{2}+2 g_{2,0} g_{0,2}+4 g_{1,0} g_{1,2}+4 g_{0,1} g_{2,1}, \\
& \tilde{g}_{1,3}=6 g_{1,1} g_{0,2}+6 g_{0,1} g_{1,2}+2 g_{1,0} g_{0,3}, \\
& \tilde{g}_{0,4}=6 g_{0,2}^{2}+8 g_{0,1} g_{0,3},
\end{aligned}
$$

The derivatives of $\hat{g}:=g(1-g)$ at $\left(x_{*}, y_{*}\right)$ up to order 2 :

$$
\begin{aligned}
& \hat{g}_{1,0}=\left(1-2 y_{*}\right) g_{1,0}, \quad \hat{g}_{0,1}=\left(1-2 y_{*}\right) g_{0,1}, \\
& \hat{g}_{2,0}=-2 g_{1,0}^{2}+\left(1-2 y_{*}\right) g_{2,0}, \\
& \hat{g}_{1,1}=-2 g_{1,0} g_{0,1}+\left(1-2 y_{*}\right) g_{1,1}, \\
& \hat{g}_{0,2}=-2 g_{0,1}^{2}+\left(1-2 y_{*}\right) g_{0,2} .
\end{aligned}
$$

Acknowledgements. The authors thank Emanuele Mingione for many useful discussions in particular those about the integral representation of the partition function. DA acknoledges financial support from Progetto Giovani 2017 GNFM Indam. PC acknowledges financial support from PRIN (grant number 2010HXAW77). CV acknowledges financial support from Fondo di Ateneo per la Ricerca 2017 (UniMoRe).

\section{References}

[1] D. Alberici, "A cluster expansion approach to the Heilmann-Lieb liquid crystal model", Journal of Statistical Physics 162, 761-791 (2016)

[2] D. Alberici, P. Contucci, "Solution of the monomer-dimer model on locally tree-like graphs. Rigorous results", Communications in Mathematical Physics 331(3), 975-1003 (2014)

[3] D. Alberici, P. Contucci, E. Mingione, "A mean-field monomer-dimer model with attractive interaction: exact solution and rigorous results", Journal of Mathematical Physics 55(6), 063301 (2014)

[4] D. Alberici, P. Contucci, E. Mingione, "The exact solution of a meanfield monomer-dimer model with attractive potential", EuroPhysics Letters 106(1), 10001 (2014)

[5] D. Alberici, P. Contucci, E. Mingione, "A mean-field monomer-dimer model with randomness: exact solution and rigorous results", Journal of Statistical Physics 160(6), 1721-1732 (2015)

[6] D. Alberici, P. Contucci, M. Fedele, E. Mingione, "Limit theorems for monomer-dimer mean-field models with attractive potential", Communications in Mathematical Physics 346(3), 781-799 (2016) 
[7] D. Alberici, P. Contucci, E. Mingione, "Non-Gaussian fluctuations in monomer-dimer models", EuroPhysics Letters 114(1), 10006 (2016)

[8] E. Agliari, A. Barra, P. Contucci, A. Pizzoferrato, C. Vernia, "Social interaction effects on immigrant integration", Palgrave Communications 4:55 (2018)

[9] E. Agliari, A. Barra, L. Dello Schiavo, A. Moro, "Complete integrability of information processing by biochemical reactions", Scientific Reports 6:36314 (2016)

[10] A. Barra, P. Contucci, R. Sandell, C. Vernia, "An analysis of a large dataset on immigrant integration in Spain. The Statistical Mechanics perspective on Social Action", Scientific Reports 4, 4174 (2014)

[11] C. Bordenave, M. Lelarge, J. Salez, "Matchings on infinite graphs", Probability Theory and Related Fields 157, 183-208 (2013)

[12] N.G. De Bruijn, Asymptotic methods in Analysis, North-Holland, 1961

[13] W.-K. Chen, "Limit theorems in the imitative monomer-dimer meanfield model via Stein's method", Journal of Mathematical Physics 57(8), 083302 (2016)

[14] P. Contucci, M. Degli Esposti, C. Giardinà, S. Graffi, "Thermodynamical limit for correlated Gaussian Random Energy Models", Communications in Mathematical Physics 236, 55-63 (2003)

[15] P. Contucci, S. Graffi, "On the Surface Pressure for the EdwardsAnderson Model", Communications in Mathematical Physics 248(1), 207-216 (2004)

[16] P. Contucci, R. Luzi, C. Vernia, "Inverse problem for the mean-field monomer-dimer model with attractive interaction", Journal of Physics A: Mathematical and Theoretical 50(20), 205002 (2017)

[17] P. Contucci, S. Graffi, "On the Surface-Pressure for the EdwardsAnderson Model", Communications in Mathematical Physics 248, 207216 (2004)

[18] P. Contucci, J. Lebowitz, "Correlation Inequalities for Spin Glasses", Annales Henri Poincaré 8, 1461-1467 (2007)

[19] M.E. Fisher, G. Caginalp, "Wall and boundary free energies", Communications in Mathematical Physics 56(1), 11-56 (1977)

[20] R. B. Griffiths, "Correlation in Ising ferromagnets", Journal of Mathematical Physics 8, 478-483 (1967)

[21] R. B. Griffiths, "A proof that the free energy of a spin system is extensive", Journal of Mathematical Physics 5, 1215-1222 (1964)

[22] O.J. Heilmann, E.H. Lieb, "Theory of monomer-dimer systems", Communications in Mathematical Physics 25, 190-232 (1972)

[23] O.J. Heilmann, E.H. Lieb, "Monomers and dimers", Physical Review Letters 24, 1412-1414 (1970)

[24] O.J. Heilmann, E.H. Lieb, "Lattice models for liquid crystals", Journal of Statistical Physics 20, 680-693 (1979) 
[25] I. Jauslin, E.H. Lieb, "Nematic liquid crystal phase in a system of interacting dimers and monomers", Communications in Mathematical Physics 363(3), 955-1002 (2018)

[26] D.G. Kelly, S. Sherman, "General Griffiths' inequalities on correlations in Ising ferromagnets", Journal of Mathematical Physics 9, 466 (1968)

[27] Q.-M. Shao, M. Zhang, Z.-S. Zhang, "Cramér-type moderate deviation theorems for nonnormal approximation", preprint https://arxiv.org/abs/1809.07966

[28] I.G. Vladimirov, "The monomer-dimer problem and moment Lyapunov exponents of homogeneous Gaussian random fields", Discrete and Continuous Dynamical Systems B 18(2), 575-600 (2013)

[29] L. Zdeborová, M. Mézard, "The number of matchings in random graphs", Journal of Statistical Mechanics 5, P05003 (2006) 